\title{
Cardiovascular magnetic resonance and PET-CT of left atrial paraganglioma
}

\author{
Anderanik Tomasiann ${ }^{1}$, Chi Lai ${ }^{2}$, Stefan Ruehm¹, Mayil S Krishnam"
}

\begin{abstract}
Cardiac paragangliomas are among the rarest primary cardiac tumors. We present a case of left atrial paraganglioma in a patient who presented with symptoms and signs of catecholamine excess in which cardiovascular magnetic resonance in multiple orientations and PET-CT played an important role in the diagnosis and tissue characterization.
\end{abstract}

\section{Introduction}

Pheochromocytomas or functioning paragangliomas are catecholamine-producing tumors arising from secretory chromograffin cells of neuroectodermal origin. These lesions commonly originate from the adrenal medulla. Approximately $18 \%$ of pheochromocytomas are extraadrenal [1].Primary cardiac paragangliomas are extremely rare with less than 50 cases reported in the literature [2]. Recent advances in cross sectional imaging have provided the potential for non-invasive and accurate diagnosis of these lesions [3-5]. In this report, we describe a left atrial paraganglioma, characterized in a multi-modality setting and confirmed on histopathology.

\section{Case report}

A 25-year-old female in her third trimester of pregnancy, presented with paroxysmal headache, palpitations, sweating, and hypertension of up to 230/130 for three weeks. Her medical history was negative for previous hypertension, and physical examination was unremarkable. Initial work-up for hypertension excluded pre-eclampsia. Further biochemistry evaluation revealed markedly elevated urine catecholamines;

Dopamine $607 \mu \mathrm{g} / 24$ hours (range 65 - 400), Norepinephrine $978 \mu \mathrm{g} / 24$ hours (range 15 - 80), Metanephrine $108 \mu \mathrm{g} / 24$ hours (range 24 - 96), Normetanephrine 4067 $\mu \mathrm{g} / 24$ hours (range 75 - 375), and Vanillyl mandelic acid $13.6 \mathrm{mg} / 24$ hours (range 2 - 7). Epinephrine level was 0.8 $\mu \mathrm{g} / 24$ hours (range $0.0-20.0$ ). A diagnosis of pheochromocytoma was considered and the patient underwent

* Correspondence: mkrishnam@mednet.ucla.edu

${ }^{1}$ Department of Radiological Sciences, University of California at Los Angeles, USA

abdominal magnetic resonance imaging (MRI) which demonstrated a $28 \times 19$ mm left juxta-adrenal mass. Treatment with phenoxybenzamine (10 $\mathrm{mg}$, twice a day) was initiated. Six weeks following uneventful delivery by Cesarean Section, the patient underwent left laparoscopic adrenalectomy for suspected pheochromocytoma. Histopathology showed normal adrenal gland and brown fat in the juxta-adrenal mass. The patient's symptoms continued, and with the suspicion of extra-adrenal pheochromocytoma, $\mathrm{F}^{18}$ Levo-DOPA positron emission tomographycomputed tomography or PET-CT scan of the whole body was performed to localize the lesion (Siemens Medical Solutions, Erlangen, Germany). The intravenous $\mathrm{F}^{18}$ LevoDOPA dose was $6.9 \mathrm{mCi}$, and the patient was pre-medicated with $200 \mathrm{mg}$ of carbidopa 45 minutes before the scan to enhance the uptake of $\mathrm{F}^{18}$ Levo-DOPA. On PET$\mathrm{CT}$, a large hyper-metabolic soft tissue mass measuring $4.5 \times 2.7 \times 3.9 \mathrm{~cm}$ in the region of posterior mediastinum near the left atrium was noted (Figure 1, arrow).

Four days following the PET-CT examination, cardiovascular MR (CMR) was performed on a 1.5 Tesla MR scanner (Magnetom Avanto, Siemens Medical Solutions, Malvern, Pennsylvania, USA) to further characterize the lesion and its anatomical relation to the atria and surrounding structures. On Steady State Free Precession (SSFP) breath-hold cine MRI (TE: $1.2 \mathrm{~ms}$, TR: $2.4 \mathrm{~ms}$, temporal resolution: $40.3 \mathrm{~ms}$ ), the epicenter of the hypo-intense soft tissue mass was noted to be in the posterior mediastinum with no apparent fat plane between the lesion and the posterior wall of the left atrium (Figure 2-a, arrow). The mass caused smooth extrinsic compression of left atrial wall and demonstrated typical low signal on T1-weighted spin-echo (TE: 


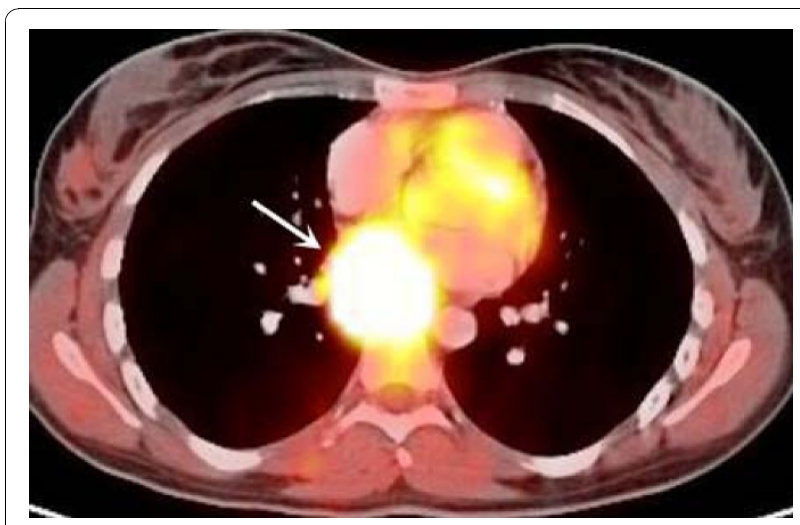

Figure 1 Axial view of PET-CT scan image demonstrates a large hypermetabolic mass with intense uptake of $\mathrm{F}^{18}$ LevoDOPA in the posterior mediastinum near the left atrium which is consistent with paraganglioma.

$14 \mathrm{~ms}$, TR: $750 \mathrm{~ms}$ ) and high signal on T2-weighted dark blood turbo spin-echo (TE: $86 \mathrm{~ms}$, TR: $4000 \mathrm{~ms}$ ) images (Figure 2-b).

During administration of $5 \mathrm{~mL}$ of gadopentetate dimeglumine (Magnevist, Berlex Laboratories, Wayne, NJ), T1-weighted inversion recovery gradient-echo myocardial perfusion imaging was performed which demonstrated heterogeneous dynamic contrast filling of the mass (Figure 2-c). In addition, 10 minutes following injection of 0.2 $\mathrm{mmol} / \mathrm{kg}$ body weight of Magnevist, late gadolinium enhancement (LGE) CMR was performed using T1weighted, fat-saturated, inversion recovery sequence. This demonstrated peripheral rim enhancement of the mass with central dark signal suggestive of central tissue necrosis (Figure 2-d). Based on clinical history, urine biochemistry, and radiological features, a diagnosis of cardiac paraganglioma, although very rare, was considered. Surgical procedure was performed through a right anterolateral thoracotomy, and following cardio-pulmonary bypass, the tumor was excised. At surgery, the left atrial mural mass was $4.5 \mathrm{~cm}$ in largest dimension originating in the posterior wall with extension to Sondergaard's groove and interatrial septum. Following excision, left atrial wall and pericardial reconstructions were performed using bovine pericardium and Gore-Tex patch, respectively. Two days after surgery, 24-hour urine catecholamines returned to normal range, and following uneventful recovery, the patient was discharged in stable condition. Histopathologic evaluation indeed confirmed a cardiac paraganglioma (Figure 3).

The patient remained asymptomatic during the 4month follow-up period without local recurrence on echocardiography.

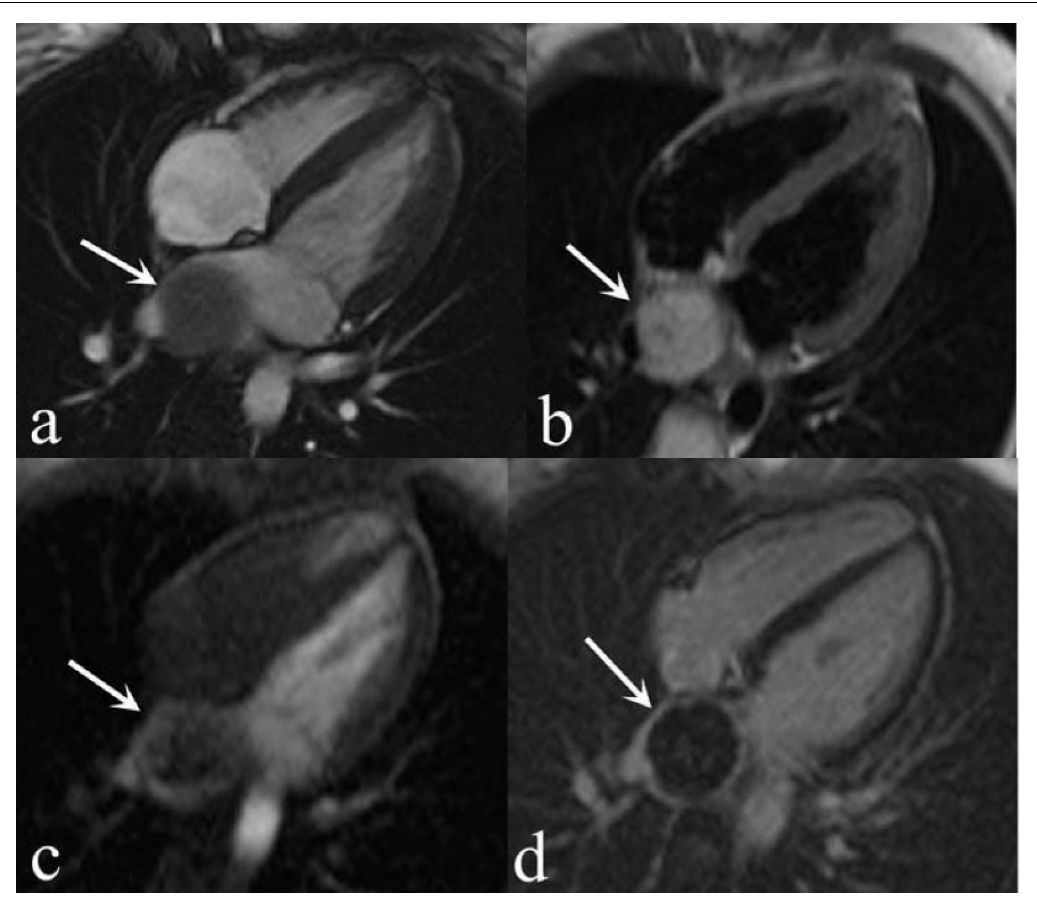

Figure 2 Horizontal long axis CMR images demonstrate a round, well defined posterior mediastinal mass with smooth extrinsic compression of left atrial wall which is hypo-intense on breath-hold Steady State Free Precession (SSFP) cine CMR ( $a$, arrow), and shows homogenously high signal on T2-weighted dark blood turbo spin-echo image (b, arrow). T1-weighted inversion recovery gradientecho myocardial perfusion imaging demonstrates heterogeneous, mostly peripheral, dynamic contrast filling of the mass suggesting vascularity (c, arrow), and T1-weighted, fat-saturated, inversion recovery late gadolinium enhancement image shows peripheral rim enhancement with no evidence of central enhancement suggestive of central tissue necrosis (d, arrow). 


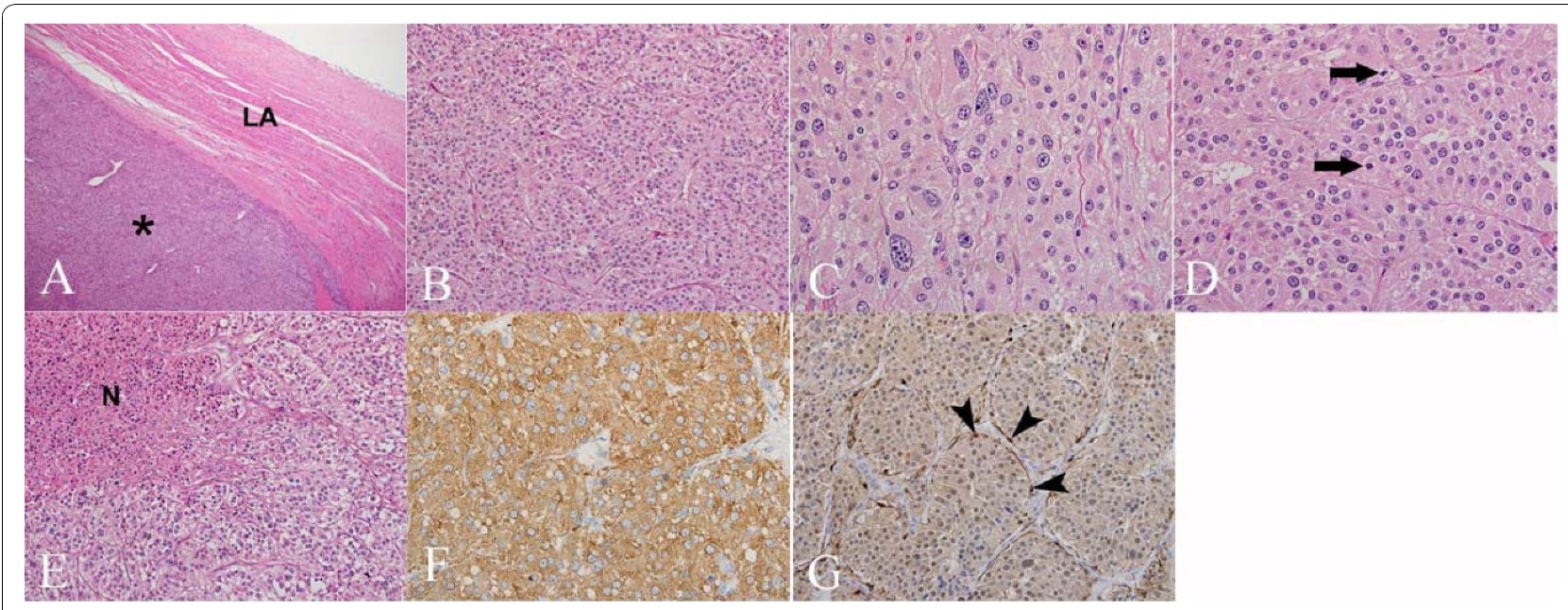

Figure 3 (A) Low power photomicrograph (40x; H\&E stain) demonstrates tumor $\left(^{*}\right)$ compressing left atrium (LA). (B) The tumor is comprised of nests of fairly uniform cells with moderate eosinophilic, granular cytoplasm (200x; H\&E stain). (C) Marked cytologic atypia with pleomorphic nuclei and prominent nucleoli was focally present (400x; H\&E stain) in addition to (D) increased number of mitoses (arrows) (400x; H\&E stain). (E) Scattered foci of coagulative tumor necrosis (N) were also noted (200x; H\&E stain). (F) The tumor exhibited diffuse, strong cytoplasmic positivity for synaptophysin immunostain (400X). (G) S100 immunostain highlights sustentacular cells surrounding nests of tumor cells $(400 x)$.

\section{Discussion}

Primary cardiac tumors are rare with a reported prevalence of $0.001 \%$ to $0.03 \%$ on autopsy series [6]. Of these tumors, cardiac paragangliomas are among the rarest accounting for less than $1 \%$ of cases [6]. These tumors originate from paraganglial cells of the arteries or the visceral autonomic paraganglia of the atria [7]. Cardiac paragangliomas are most commonly located in the left atrium [1] and less frequent sites include right atrium, inter-atrial septum, and the left ventricle $[1,4,8]$. The mean age at the presentation is $36-40$ years with approximately equal sex distribution [9]. Cardiac paragangliomas are catecholeamine-secreting in $35-50 \%$ of cases [6].

Clinical presentations of cardiac paraganglioma range from an incidental finding [9] to constitutional symptoms including malaise, weight loss and fever [3], symptoms and signs of catecholamine excess (paroxysmal headache, palpitation, sweating, and hypertension) [1], arrhythmia due to invasion of the conductive system [4], angina pectoris or myocardial infarction secondary to compression or obstruction of coronary arteries [5], acute heart failure or dilated cardiomyopathy [10], pericardial involvement, and disruption of valvular function [4].

The incidence of malignancy is reported to be approximately $10 \%$ [6]. However, there are no histological criteria to differentiate benign from malignant cardiac paragangliomas [11]. Malignancy is determined by tumor behavior rather than histological appearance and the distinguishing feature includes presence of distant metastasis [11]. Histopathologically, paragangliomas are monomorphous tumors composed of nests of paraganglial cells (Zellballen) surrounded by sustentacular cells [12].

Cardiac paragangliomas are echogenic masses on echocardiography and are reported as circumscribed, heterogeneous masses with low attenuation on unenhanced CT [14] with marked enhancement on contrast enhanced CT [12].

These lesions can be localized using very specific radiotracers such as ${ }^{131} \mathrm{I}$ or ${ }^{123} \mathrm{I}$ metaiodobenzylguanidine or ${ }^{18} \mathrm{~F}$-DOPA, which are actively transported into neurosecretory granules of catecholamine-producing cells [13]. ${ }^{18} \mathrm{~F}$-DOPA PET studies have yielded promising results in the imaging of pheochromocytoma [13], demonstrating typical intense uptake of ${ }^{18} \mathrm{~F}$-DOPA by the paraganglional cells of the lesions, as showed in our case.

Due to its higher spatial resolution and multiplanar image acquisition, CMR is considered an important technique in diagnosis and characterization of cardiac tumors. Cine SSFP and pre-contrast T1- and T2weighted imaging are useful for evaluation of anatomic features, whereas first pass dynamic perfusion and late gadolinium enhancement sequences are important in assessment of vascularity.

As seen in our patient, cardiac paragangliomas are typically iso- or hypo-intense relative to myocardium on T1-weighted, and markedly hyper-intense on T2weighted images [14]. They are highly vascular and as in our case, may typically demonstrate dynamic contrast filling on myocardial perfusion imaging which is often 
heterogeneous, with central non-enhancing areas suggesting tumor necrosis [14]. Presence of peripheral rim enhancement on LGE imaging also indicates vascularity of these lesions distinguishing them from avascular cardiac masses such as thrombi and lipoma. Although presence of central dark signal on LGE could be due to nulling of signal following Inversion pulse, it likely represents tissue necrosis. Cardiac hemangiomas demonstrate LGE, intermediate and high signal intensity on T1 and T2-weighted images, respectively, and show enhancement on perfusion imaging [12]. Cardiac lymphangiomas demonstrate high signal intensity on $\mathrm{T} 1$ and $\mathrm{T} 2$ weighted images, whereas rhabdomyomas are isointense on T1 and hyper-intense on T2 weighted images compared to adjacent myocardium [14].

Surgical resection of cardiac paraganglioma results in complete cure and relief of symptoms [9,12]. Surgical risks particular to these lesions include fatal hemorrhage due to high vascularity, and hypertensive crisis from intra-operative manipulation which may be avoided by cardiopulmonary bypass allowing safe dissection. Since paragangliomas may be infiltrative lesions, as in our patient, extensive resection of the atrial wall may be required for complete excision [7].

\section{Conclusion}

The combination of CMR with various image acquisition sequences in multiple orientations and PET-CT studies, provides a powerful tool for non-invasive morphological assessment and tissue characterization of cardiac paraganglioma, especially when correlated with clinical history and appropriate biochemistry evaluations.

\section{Consent}

Written informed consent was obtained from the patient for publication of this case report and any accompanying images. A copy of the written consent is available for review by the Editor-in-Chief of this journal.

\section{Author details}

'Department of Radiological Sciences, University of California at Los Angeles, USA. ${ }^{2}$ Department of Pathology, University of California at Los Angeles, USA.

\section{Authors' contributions}

All authors were involved in the conception and design of the manuscript, acquisition of data, drafting the manuscript, critical revision of the manuscript for intellectual content, and final approval.

\section{Competing interests}

The authors declare that they have no competing interests.

Received: 17 June 2009

Accepted: 4 January 2010 Published: 4 January 2010
References

1. Manger WM: An overview of pheochromocytoma: history, current concepts, vagaries, and diagnostic challenges. Ann N Y Acad Sci 2006, 1073:1-20.

2. Mandak JS, Benoit CH, Starkey RH, Nassef LAJr: Echocardiography in the evaluation of cardiac pheochromocytoma. Am Heart J 1996, 132:1063-6.

3. Turley AJ, Hunter S, Stewart MJ: A cardiac paraganglioma presenting with atypical chest pain. Eur J Cardiothorac Surg 2005, 28(2):352-4.

4. Maxey TS, Grow P, Morris CD, Patton KT, Guyton RA: Biatrial primary cardiac paraganglioma: a rare finding. Cardiovasc Pathol 2007, 16(3):17982.

5. Sawka AM, Young WFJr, Schaff HV: Cardiac phaeochromocytoma presenting with severe hypertension and chest pain. Clin Endocrinol 2001, 54(5):689-92.

6. Burke A, Virmani R: Tumors of the heart and great vessels. Atlas of tumor pathology. 3rd series, fascicle 16 Washington, DC: Armed Forces Institute of Pathology 1996.

7. Orringer MB, Sisson JC, Glazer G, et al: Surgical treatment of cardiac pheochromocytomas. J Thorac CardiovasC Surg 1985, 89:753-757.

8. Cane ME, Berrizbeitia LD, Yang SS, Mahapatro D, McGrath LB: Paraganglioma of the interatrial septum. Ann Thorac Surg 1996, 61(6):1845-7.

9. Jebara VA, Uva MS, Farge A, et al: Cardiac pheochromocytomas. Ann Thorac Surg 1992, 53(2):356-61, Review..

10. Sardesai SH, Mourant AJ, Sivathandon Y, Farrow R, Gibbons DO: Phaeochromocytoma and catecholamine induced cardiomyopathy presenting as heart failure. Br Heart J 1990, 63:234-7.

11. Nomura A, Kakinoki S, Fujita M, Kitabatake A: Images in cardiovascular medicine. Malignant cardiac pheochromocytoma with bone metastases. Circulation 1998, 97(19):1993-4.

12. Grebenc ML, Rosado de Christenson ML, Burke AP, et al: Primary Cardiac and Pericardial Neoplasms: Radiologic-Pathologic Correlation. Radiographics 2000, 20:1073-1103.

13. Hoegerle S, Nitzsche E, Altehoefer C, et al: Pheochromocytomas: detection with18F DOPA whole body PET-initial results. Radiology 2002, 222:507512

14. Araoz PA, Mulvagh SL, Tazelaar HD, Julsrud PR, Breen JE: CT and MR imaging of benign primary cardiac neoplasms with echocardiographic correlation. Radiographics 2000, 20(5):1303-1319.

doi:10.1186/1532-429X-12-1

Cite this article as: Tomasian et al:: Cardiovascular magnetic resonance and PET-CT of left atrial paraganglioma. Journal of Cardiovascular

Magnetic Resonance 2010 12:1.
Publish with Bio Med Central and every scientist can read your work free of charge

"BioMed Central will be the most significant development for disseminating the results of biomedical research in our lifetime. " Sir Paul Nurse, Cancer Research UK

Your research papers will be:

- available free of charge to the entire biomedical community

- peer reviewed and published immediately upon acceptance

- cited in PubMed and archived on PubMed Central

- yours - you keep the copyright
BioMedcentral 